\title{
Maltreatment Exposure, Brain Structure, and Fear Conditioning in Children and Adolescents
}

\author{
Katie A McLaughlin*,', Margaret A Sheridan², Andrea L Gold', Andrea Duys', Hilary K Lambert', \\ Matthew Peverill', Charlotte Heleniak', Tomer Shechner ${ }^{4}$, Zuzanna Wojcieszak ${ }^{5}$ and Daniel S Pine ${ }^{3}$ \\ 'Department of Psychology, University of Washington, Seattle, WA, USA; ' Department of Psychology, University of North Carolina, Chapel Hill, NC, \\ USA; ${ }^{3}$ Section on Development and Affective Neuroscience, National Institute of Mental Health, Bethesda, MD, USA; ${ }^{4}$ Department of Psychology, \\ University of Haifa, Haifa, Israel; ${ }^{5}$ Department of Psychology, Temple University, Philadelphia, PA, USA
}

\begin{abstract}
Alterations in learning processes and the neural circuitry that supports fear conditioning and extinction represent mechanisms through which trauma exposure might influence risk for psychopathology. Few studies examine how trauma or neural structure relates to fear conditioning in children. Children $(n=94)$ aged 6-18 years, 40.4\% $(n=38)$ with exposure to maltreatment (physical abuse, sexual abuse, or domestic violence), completed a fear conditioning paradigm utilizing blue and yellow bells as conditioned stimuli (CS+/CS - ) and an aversive alarm noise as the unconditioned stimulus. Skin conductance responses (SCR) and self-reported fear were acquired. Magnetic resonance imaging data were acquired from 60 children. Children without maltreatment exposure exhibited strong differential conditioning to the CS+ vs CS - , based on SCR and self-reported fear. In contrast, maltreated children exhibited blunted SCR to the CS+ and failed to exhibit differential SCR to the CS+ vs CS - during early conditioning. Amygdala and hippocampal volume were reduced among children with maltreatment exposure and were negatively associated with SCR to the CS+ during early conditioning in the total sample, although these associations were negative only among non-maltreated children and were positive among maltreated children. The association of maltreatment with externalizing psychopathology was mediated by this perturbed pattern of fear conditioning. Child maltreatment is associated with failure to discriminate between threat and safety cues during fear conditioning in children. Poor threatsafety discrimination might reflect either enhanced fear generalization or a deficit in associative learning, which may in turn represent a central mechanism underlying the development of maltreatment-related externalizing psychopathology in children.

Neuropsychopharmacology (2016) 41, 1956-1964; doi:I0.1038/npp.2015.365; published online I3 January 2016
\end{abstract}

\section{INTRODUCTION}

Childhood trauma, particularly maltreatment, increases risk for psychopathology (Kilpatrick et al, 2003; McLaughlin et al, 2013). Leading theories suggest that psychopathology following trauma arises through perturbed fear conditioning and associated disruptions in neural circuitry underlying the acquisition and extinction of fear (Jovanovich and Ressler, 2010; Lissek and van Meurs, 2015). The current study examines relationships among maltreatment, fear conditioning, brain structure, and psychopathology in children.

Traumatic events occurring early in development are likely to influence fear conditioning. Children frequently develop fears of both real and imagined threats as they learn to distinguish between threat and safety cues in their environment. Developmental variation in fear conditioningparticularly, extinction learning-occurs in rodents (Kim and Richardson, 2010; Patwell et al, 2012) and humans (Glenn et al, 2012; Shechner et al, 2014b). In rodents,

\footnotetext{
*Correspondence: Dr KA McLaughlin, Department of Psychology, University of Washington, Box 351525, Seattle, WA 98195, USA, Tel: + | 206 616 7863, Fax: + | 206685 3157, E-mail: mclaughk@uw.edu Received I October 2015; revised 19 November 2015; accepted I5 December 20।5; accepted article preview online 18 December 2015
}

exposure to threatening early environments alters fear conditioning, resulting in enhanced acquisition and reduced extinction of fear (Matsumoto et al, 2008; Toledo-Rodriguez and Sandi, 2007). Moreover, maltreated children exhibit lasting alterations in threat processing, including heightened attention and greater neural responses to potential threats (McCrory et al, 2011; McLaughlin et al, 2015; Pollak and Tolley-Schell, 2003). Although conceptual models argue that child maltreatment leads to lasting alterations in fear conditioning (McLaughlin et al, 2014b; Sheridan and McLaughlin, 2014), surprisingly little research has examined this possibility in children. We do so in the current study.

The neural circuitry underlying fear conditioning is conserved across species (Johansen et al, 2011; Kim and Jung, 2006). Relevant brain regions include the amygdala, where information about the conditioned stimulus (CS) and unconditioned stimulus (US) converges to facilitate fear acquisition; the hippocampus and ventromedial prefrontal cortex (vmPFC), which support extinction learning and retrieval; and the dorsal anterior cingulate cortex (dACC), which is involved in fear expression. Morphology of these regions relates to individual differences in fear acquisition and extinction; amygdala volume is negatively associated with fear acquisition in rodents and human adults 
(Hartley et al, 2011; Yang et al, 2008). dACC thickness is associated with enhanced fear acquisition (Milad et al, 2007), whereas vmPFC thickness is associated with retention of extinction learning in humans (Hartley et al, 2011; Milad et al, 2005). Reduced amygdala and hippocampal volume and vmPFC thickness and volume have been observed among maltreated children (Hanson et al, 2010, 2015), which may contribute to alterations in fear conditioning following maltreatment. We are unaware of previous studies examining the link between neural structure and fear conditioning in children.

Together, disruptions in fear conditioning and underlying neural circuitry might represent a pathway leading to heightened psychopathology among children who have experienced maltreatment. Atypical fear conditioning has been observed in multiple forms of psychopathology. Posttraumatic stress disorder (PTSD) is characterized by failure to maintain extinction learning (Milad et al, 2009) and inhibit the fear memory, even in the presence of safety cues (Jovanovich and Norrholm, 2011; Jovanovich and Ressler, 2010). Anxiety disorders are associated with exaggerated fear responses during conditioning and extinction learning (Craske et al, 2008; Lau et al, 2008). In contrast, externalizing psychopathology is associated with blunted fear conditioning and poor discrimination between threat and safety cues (Fairchild et al, 2008). Examining fear conditioning in maltreated children might clarify how fear conditioning contributes to trauma-related psychopathology.

The current study examines how maltreatment relates to fear conditioning in children, with the hypothesis that maltreatment would be associated with impaired extinction learning. In addition, we examine associations of fearlearning indices with the structure of brain regions involved in fear conditioning to determine whether associations in children mirror those in adults. Finally, we determine whether alterations in fear conditioning are a mechanism linking maltreatment with psychopathology in children.

\section{MATERIALS AND METHODS}

\section{Sample}

The sample included 94 youths aged 6-18 years who were recruited at schools, after-school and prevention programs, medical clinics, and the general community in Seattle, WA between February 2014 and February 2015. Recruitment efforts aimed to recruit a sample with variation in maltreatment exposure. To do so, we recruited from neighborhoods with high levels of violent crime, from clinics that served a predominantly low-SES catchment area, and agencies that work with families who have experienced violence (eg, domestic violence shelters, programs for parents mandated to receive intervention by Child Protective Services). See Table 1 for socio-demographic characteristics of the sample.

A subset of 60 participants $(n=24$ with maltreatment exposure, $n=36$ age- and gender-matched controls) completed an MRI scan during a separate visit. Distributions of socio-demographic variables were nearly identical to the larger sample. Procedures were approved by the Institutional Review Board at the University of Washington. Written informed consent was obtained from legal guardians; children provided written assent.

\section{Procedure}

Participants completed a fear conditioning task validated for children (Supplementary Figure S1; Shechner et al, 2014a). Images of a blue and yellow bell were used as the CS+ and CS - , counter-balanced across participants. The US was an aversive $95 \mathrm{~dB}$ alarm noise. The task involved three phases: preconditioning, conditioning, and extinction. During preconditioning, participants viewed the CS+ and CS - in the absence of the US (four trials each). The conditioning phase involved 10 trials each of the CS+ and CS - ; the CS+ co-terminated with the US in $80 \%$ of trials. During extinction, the CS+ and CS - were presented in the absence of the US (eight trials each). The inter-trial interval ranged from 8 to $12 \mathrm{~s}$ (mean $=10 \mathrm{~s}$ ). Participants reported how much they feared, liked, and found the CS+ and CSunpleasant after each phase using a Likert scale ranging from 0 (none) to 10 (extreme). These ratings were summed (with ratings for liking reverse-scored) to create a self-reported fear composite for each phase.

Equipment malfunctions resulted in loss of physiological data from two participants, one participant declined to complete the task, and one participant discontinued the task during conditioning. These participants were excluded from analysis, resulting in an analytical sample of 90 participants.

\section{Measures}

Child maltreatment. Child abuse and domestic violence were assessed using an interview, the Childhood Experiences of Care and Abuse (CECA) interview (Bifulco et al, 1997), and a self-report questionnaire, the Childhood Trauma Questionnaire (CTQ) (Bernstein et al, 1997). The CECA assesses caregiving experiences, including physical and sexual abuse; we modified the interview to ask parallel questions about witnessing domestic violence (ie, directly observing violence directed at a caregiver). Inter-rater reliability for maltreatment reports is excellent, and validation studies suggest high agreement between siblings on maltreatment reports (Bifulco et al, 1997). The CTQ is a 28-item scale that assesses the frequency of maltreatment during childhood, including physical and sexual abuse. The CTQ has good convergent and discriminant validity (Bernstein et al, 1997).

We used the CECA and the CTQ to create a dichotomous indicator of maltreatment. Participants who reported physical abuse, sexual abuse, or witnessing more than two incidents of domestic violence during the interview or who had a score on the CTQ physical or sexual abuse sub-scales above a validated threshold (Walker et al, 1999) were classified as maltreated. A total of $40.4 \%$ of the sample $(n=38)$ were maltreated. We additionally created indicators of maltreatment frequency using the CTQ sub-scales for physical and sexual abuse, and a measure of maltreatment severity reflecting the number of discrete types of maltreatment each participant experienced.

Participants in the control group had no maltreatment exposure but were not excluded for exposure to other forms of trauma, such as accidents, injuries, and witnessing community violence (assessed using the UCLA PTSD Reaction Index, described below). 
Table I Distribution of Socio-Demographics and Psychopathology by Maltreatment $(N=90)$

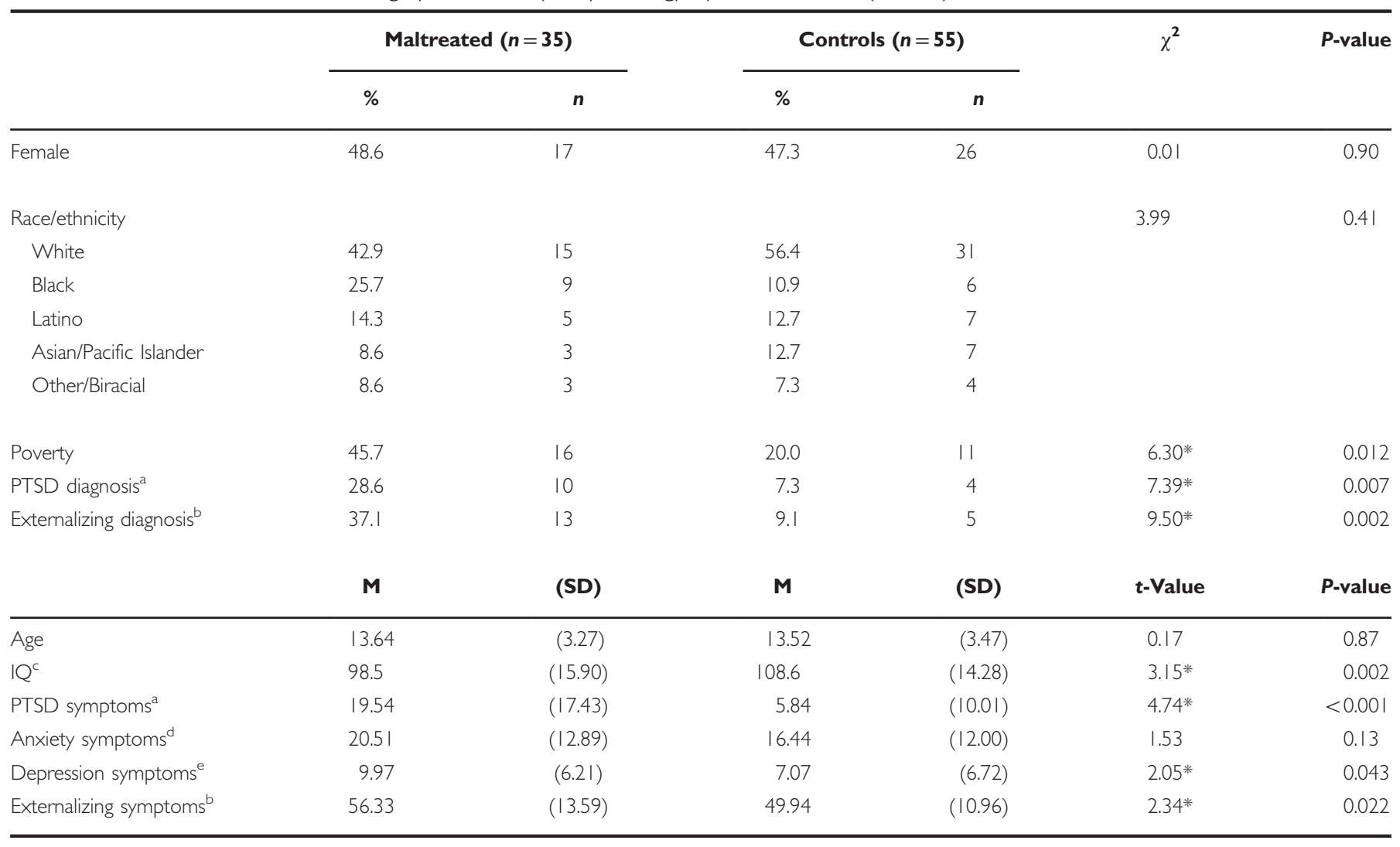

aPTSD symptoms and analog diagnosis were assessed with the UCLA PTSD Reaction Index (PTSD-RI), child report.

${ }^{b}$ Externalizing symptoms and analog diagnosis were assessed with the Child Behavior Checklist (CBCL), parent report.

${ }^{\mathrm{IQ}}$ was assessed using the Wechsler Abbreviated Scale of Intelligence (WASI).

${ }^{d}$ Anxiety symptoms were assessed with the Child Anxiety Related Emotional Disorders (SCARED), child report. Depression symptoms were assessed with the Children's Depression Inventory (CDI), child report.

$* P<0.05$, two-tailed test.

Physiological measures. Measures of electrodermal activity (EDA) were acquired continuously throughout the task and served as the primary measure of fear conditioning. EDA was obtained with a Biopac galvanic skin response module (Goleta, CA). Two Ag-AgCl electrodes filled with sodium chloride gel were attached to the distal phalanges of the index and middle finger of the non-dominant hand after the phalanges were cleaned with rubbing alcohol and abraded. The sampling rate was $250 \mathrm{~Hz}$. EDA was analyzed using the AcqKnowledge 4.0 software (Biopac Systems, Goleta, CA). Skin conductance responses (SCR) were calculated following standard procedures (Cacioppa et al, 2007) as the difference from a 1-s pre-CS baseline to peak response in the 1-4s following stimulus onset, with a minimum response of 0.02 microsiemens $(\mu s)$. Nine participants were nonresponders based on this threshold and were evenly distributed across the maltreated $(n=5)$ and control groups $(n=4)$. Non-responders were included in our analysis, which is standard in fear conditioning studies of children. The results were unchanged when non-responders were excluded.

Neural structure. Scanning was performed on a 3-T Phillips Achieva scanner at the University of Washington Integrated Brain Imaging Center using a 32-channel head coil. T1-weighted multi-echo MPRAGE volumes were acquired $\left(\mathrm{TR}=2530 \mathrm{~ms}, \mathrm{TE}=1640-7040 \mathrm{~ms}\right.$, flip angle $=7^{\circ}$, FOV $=220 \mathrm{~mm}^{2}, 176$ slices, in-plane voxel size $=1 \mathrm{~mm}^{3}$ ).

T1-weighted scans were processed using FreeSurfer version 5.3 (Fischl and Dale, 2000). Automatic image segmentation and parcellation was used to identify sub-cortical gray matter structures and estimate cortical thickness. The results were inspected and manually edited to optimize accurate placement of gray/white and gray/CSF borders. We examined volume of bilateral amygdala and hippocampus and thickness of dACC and vmPFC (bilateral caudal anterior cingulate and bilateral medial orbitofrontal cortex from the 2005 Freesurfer atlas, respectively).

Psychopathology. Symptoms of PTSD were assessed using child- and parent-report versions of the UCLA PTSD Reaction Index (PTSD-RI) (Steinberg et al, 2004). The PTSD-RI includes a 13-item trauma screen that we used to create a composite index of non-maltreatment trauma exposure and assesses PTSD re-experiencing, avoidance/ numbing, and hyper-arousal symptoms according to DSM-IV criteria (Five parents whose children did not report exposure to abuse or domestic violence indicated that their child had been exposed to some form of violence on the trauma screen; all analyses were repeated with these five 
Table 2 SCR During Each Phase of the Fear Conditioning Task as a Function of Trauma Exposure $(n=90)$

\begin{tabular}{|c|c|c|c|c|c|c|c|c|c|c|c|c|c|c|c|c|c|c|c|c|}
\hline \multirow[b]{3}{*}{ SCR } & \multicolumn{4}{|c|}{ Preconditioning } & \multicolumn{4}{|c|}{ Early conditioning } & \multicolumn{4}{|c|}{ Late conditioning } & \multicolumn{4}{|c|}{ Early extinction } & \multicolumn{4}{|c|}{ Late extinction } \\
\hline & \multicolumn{2}{|c|}{ CS+ } & \multicolumn{2}{|c|}{ CS - } & \multicolumn{2}{|c|}{ CS+ } & \multicolumn{2}{|c|}{ CS - } & \multicolumn{2}{|c|}{ CS+ } & \multicolumn{2}{|c|}{ CS - } & \multicolumn{2}{|c|}{ CS+ } & \multicolumn{2}{|c|}{ CS - } & \multicolumn{2}{|c|}{ CS+ } & \multicolumn{2}{|c|}{ CS - } \\
\hline & M & (SD) & $\mathbf{M}$ & (SD) & $\mathbf{M}$ & (SD) & $\mathbf{M}$ & (SD) & $\mathbf{M}$ & (SD) & $\mathbf{M}$ & (SD) & $\mathbf{M}$ & (SD) & $\mathbf{M}$ & (SD) & $\mathbf{M}$ & (SD) & $\mathbf{M}$ & (SD) \\
\hline Total sample & 0.11 & $(0.16)$ & 0.09 & $(0.15)$ & 0.38 & $(0.46)$ & 0.18 & $(0.37)$ & 0.25 & $(0.32)$ & 0.09 & $(0.12)$ & 0.11 & $(0.18)$ & 0.09 & $(0.14)$ & 0.08 & $(0.15)$ & 0.14 & $(0.32)$ \\
\hline
\end{tabular}

children included in the trauma group and with these five children removed from the analysis; all results were unchanged). The PTSD-RI generates a PTSD symptom severity score and an analog diagnosis and has good internal consistency and convergent validity (Steinberg et al, 2013). Internal consistency was excellent ( $\alpha=0.95$ for child and parent versions). Findings were similar for child and parent report; thus we present only child-report data.

Anxiety symptoms were assessed with child report on the Child Anxiety Related Emotional Disorders (SCARED) (Birmaher et al, 1997), which assesses anxiety disorder symptoms across five domains: panic/somatic, generalized anxiety, separation anxiety, social phobia, and school phobia. The SCARED has good psychometric properties (Birmaher et $a l, 1997)$ and had excellent reliability in our sample $(\alpha=0.92)$.

The Children's Depression Inventory (CDI) (Kovacs, 1992) is a widely used self-report measure of depressive symptoms in children and adolescents. The CDI has good psychometric properties (Kovacs, 1992). The item pertaining to suicidal ideation was removed. The remaining items were summed to create a total score. The CDI demonstrated good reliability in this sample $(\alpha=0.87)$.

Externalizing symptoms were reported by parents on the Child Behavior Checklist (CBCL) (Achenbach, 1991). The CBCL scales are among the most widely used measures of youth emotional and behavioral problems and use extensive normative data to generate age-standardized estimates of symptom severity.

\section{Data Analysis}

Average SCR was computed during five phases of the task (preconditioning; early conditioning, trials 1-10; late conditioning, trials 11-20; early extinction, trials 1-8; and late extinction, trials 9-16). A square-root transformation was performed on SCR prior to analysis. To examine SCR as a function of task conditions, we conducted a $5 \times 2$ repeated-measures ANOVA with Phase (preconditioning, early conditioning, late conditioning, early extinction, late extinction) and Stimulus (CS+, CS - ) as within-subjects factors. Self-reported fear to each stimulus was measured after preconditioning, conditioning, and extinction. A $3 \times 2$ repeated-measures ANOVA with Phase and Stimulus as within-subjects factors was conducted to examine self-reported fear in response to task conditions.

We added between-subjects factors for maltreatment and psychopathology in models for SCR and self-reported fear. Age and sex were included as covariates.
Following previous research (Milad et al, 2005), linear regression was conducted to examine associations of neural structure (ie, amygdala and hippocampal volume; thickness of dACC and vmPFC) with SCR to the CS+ and CS - during early and late conditioning and extinction. Age, sex, maltreatment, and total brain volume (for amygdala and hippocampus models only) were covariates.

Finally, we examined whether the association of maltreatment with psychopathology was explained by variation in fear conditioning using standard tests of statistical mediation. We tested the significance of indirect effects using a bootstrapping approach that provides confidence intervals for the indirect effect (Hayes, 2013).

\section{RESULTS}

\section{Fear Conditioning}

Fear conditioning occurred in the total sample. For SCR, we observed main effects of Stimulus, $F(1,356)=30.92$, $P<0.001$, with higher SCR to CS+ than $\mathrm{CS}-$, and Phase, $\mathrm{F}(4,356)=21.68, P<0.001$, with higher SCR during early and late conditioning than preconditioning or either phase of extinction, and a Stimulus $\times$ Phase interaction, $\mathrm{F}(4,356)=11.12, P<0.001$ (Table 2). Differences in SCR to the $\mathrm{CS}+$ relative to $\mathrm{CS}$ - were observed only during early and late conditioning $(P<0.001)$. Age was unrelated to fear conditioning when examined continuously, $\mathrm{F}(4,348)=1.13$, $P=0.35$, or when children (6-12 years) were compared with adolescents $(13-18$ years), $\mathrm{F}(4,348)=1.54, \quad P=0.19$ (see Supplementary Figure S2).

For self-reported fear, main effects were found for Stimulus, $\mathrm{F}(1,174)=126.19, P<0.001$, with higher self-reported fear to the CS+ than $\mathrm{CS}-$, and Phase, $\mathrm{F}(1,174)=38.28, P<0.001$, with greater self-reported fear during acquisition than preconditioning or extinction, and a Stimulus $\times$ Phase interaction, $\mathrm{F}(2,174)=70.27, \quad P<0.001 \quad$ (Table 3). Differences in selfreported fear to the $\mathrm{CS}+$ relative to $\mathrm{CS}-$ were observed following conditioning and extinction $(P<0.001)$ but not preconditioning. Age was unrelated to fear conditioning when examined continuously, $\mathrm{F}(2,170)=2.73, P=0.068$, or categorically, $\mathrm{F}(2,170)=2.42, P=0.092$ (see Supplementary Figure S3).

\section{Maltreatment and Fear Conditioning}

We next examined associations with maltreatment. The model for SCR revealed a three-way Maltreatment-by-Stimulus-byPhase interaction, $\mathrm{F}(4,344)=3.19, P=0.014$. We examined 
Table 3 Self-Reported Fear During Each Phase of the Fear Conditioning Task as a Function of Trauma Exposure $(n=90)$

\begin{tabular}{|c|c|c|c|c|c|c|c|c|c|c|c|c|}
\hline \multirow[b]{3}{*}{ Self-reported fear ${ }^{a}$} & \multicolumn{4}{|c|}{ Preconditioning } & \multicolumn{4}{|c|}{ Conditioning } & \multicolumn{4}{|c|}{ Extinction } \\
\hline & \multicolumn{2}{|c|}{ CS+ } & \multicolumn{2}{|c|}{ CS - } & \multicolumn{2}{|c|}{ CS+ } & \multicolumn{2}{|c|}{ CS - } & \multicolumn{2}{|c|}{ CS+ } & \multicolumn{2}{|c|}{ CS - } \\
\hline & $M$ & (SD) & M & (SD) & $M$ & (SD) & $\mathbf{M}$ & (SD) & $M$ & (SD) & $M$ & (SD) \\
\hline Total sample & 9.40 & $(4.47)$ & 8.60 & $(4.32)$ & 19.27 & $(6.98)$ & 7.66 & $(4.67)$ & 12.03 & $(5.65)$ & 8.59 & $(4.37)$ \\
\hline
\end{tabular}

${ }^{a}$ Self-reported fear is a composite of three items reflecting how much participants feared, liked (reverse-scored), and found the CS+ and CS - unpleasant after each phase of the task using a Likert scale ranging from 0 (none) to 10 (extreme).

fear conditioning separately for children with and without maltreatment exposure. Children without maltreatment exposure exhibited robust conditioning, with main effects of Stimulus, Phase, and a Stimulus $\times$ Phase interaction, $(P<0.001) \quad$ (Figure 1a). In contrast, maltreated children exhibited effects of Phase, $\mathrm{F}(4,136)=3.39, P=0.011$, but not Stimulus, $F(1,136)=3.85, P=0.058$, nor a Stimulus $\times$ Phase interaction, $\mathrm{F}(4,136)=0.87, P=0.49$ (Figure $1 \mathrm{~b}$ ). Maltreated children exhibited blunted SCR to the CS+ during conditioning, a similar increase in SCR to both the CS+ and CS - during early conditioning, and a differential SCR only during late conditioning. We observed a similar pattern using measures of maltreatment severity (Maltreatment-byStimulus-by-Phase interaction: $\mathrm{F}(4,340)=4.40, P=0.002$ ) and frequency of physical abuse, $\mathrm{F}(4,344)=2.39, P=0.050$ but not sexual abuse, $\mathrm{F}(4,344)=0.72, P=0.58$.

Associations of maltreatment with fear conditioning did not vary by age or sex (all $P>0.89$ ).

Maltreatment continued to predict SCR during fear conditioning after adjustment for a range of potential confounders. The three-way Maltreatment-by-Stimulus-byPhase interaction persisted after controlling for IQ, poverty, non-maltreatment trauma exposure (eg, accidents, injuries, community violence), symptoms of anxiety and depression, analog PTSD diagnosis, PTSD symptoms, and externalizing problems, $(\mathrm{F}=2.37-3.12, P=0.053-0.014)$.

Maltreatment was not associated with self-reported fear during conditioning in any model.

\section{Maltreatment and Neural Structure}

Maltreatment was associated with reduced amygdala and hippocampal volume and total brain volume but not thickness of dACC or vmPFC (Table 4).

\section{Neural Structure and Fear Conditioning}

Next we examined associations between neural structure and fear conditioning. Amygdala and hippocampus volume were negatively associated with SCR to the CS+ during early conditioning (Supplementary Table S1). Amygdala volume was negatively associated with self-reported fear to the CS+ following conditioning and vmPFC thickness was positively associated with self-reported fear to the CS - following conditioning (Supplementary Table S2).
We evaluated whether the association of amygdala and hippocampus volume with response to the CS+ during early conditioning varied as a function of maltreatment. Although the interactions of maltreatment with neural structure were not significant, visual inspection suggested that the association of amygdala and hippocampal volume with SCR to the $\mathrm{CS}+$ during early conditioning was negative among children without maltreatment histories but positive among maltreated children (Supplementary Figure S4).

\section{Fear Conditioning and Psychopathology}

Next we examined associations of fear conditioning with psychopathology. Symptoms of anxiety and depression were unrelated to fear conditioning. PTSD, $\mathrm{F}(4,344)=2.44$, $P=0.047$, and externalizing psychopathology, $\mathrm{F}(4,308)=$ $2.98, P=0.019$, were associated with SCR (ie, a Group-byStimulus-by-Phase interaction). No such associations emerged for self-reported fear. Children without PTSD exhibited greater SCR to the CS+ relative to CS - during early and late conditioning, with significant main effects of Stimulus and Phase and a Stimulus $\times$ Phase interaction $(P<0.001)$. The same pattern was observed for children without externalizing psychopathology $(P<0.001)$. In contrast, children with PTSD exhibited no effects of Stimulus, $\mathrm{F}(1,52)=1.52, P=0.24$, Phase, $\mathrm{F}(4,52)=2.45, P=0.058$, or a Stimulus $\times$ Phase interaction, $F(4,52)=1.16, P=0.34$, indicating an absence of conditioning. Similarly, children with externalizing psychopathology exhibited no effects of Stimulus, $\mathrm{F}(1,68)=0.71, P=0.41$, Phase, $\mathrm{F}(4,68)=1.90, P=0.12$, or a Stimulus $\times$ Phase interaction, $\mathrm{F}(4,68)=0.21, P=0.93$.

Critically, although maltreatment continued to be associated with fear conditioning after adjustment for PTSD and externalizing problems, PTSD, $\mathrm{F}(4,340)=2.09, P=0.082$, and externalizing psychopathology, $\mathrm{F}(4,304)=2.03, P=0.09$, were not significantly associated with fear conditioning after adjustment for maltreatment.

Finally, we evaluated whether the associations of maltreatment with PTSD and externalizing psychopathology were mediated by blunted SCR to the CS+ during early conditioning. The indirect effect of maltreatment on PTSD through fear conditioning was not significant. However, a significant indirect effect of maltreatment on externalizing psychopathology was observed through SCR during early fear conditioning ( $95 \% \mathrm{CI}: 0.04,2.58)$. The indirect effect was also significant for frequency of physical abuse (95\% CI: 0.02, 
a

Maltreated

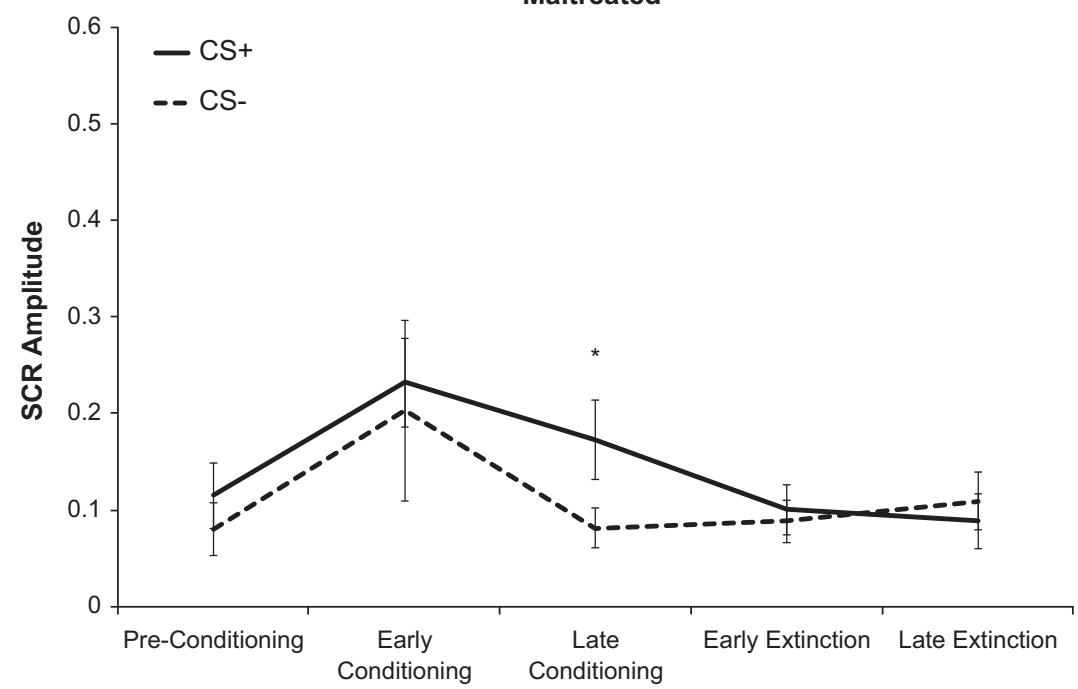

b No Maltreatment

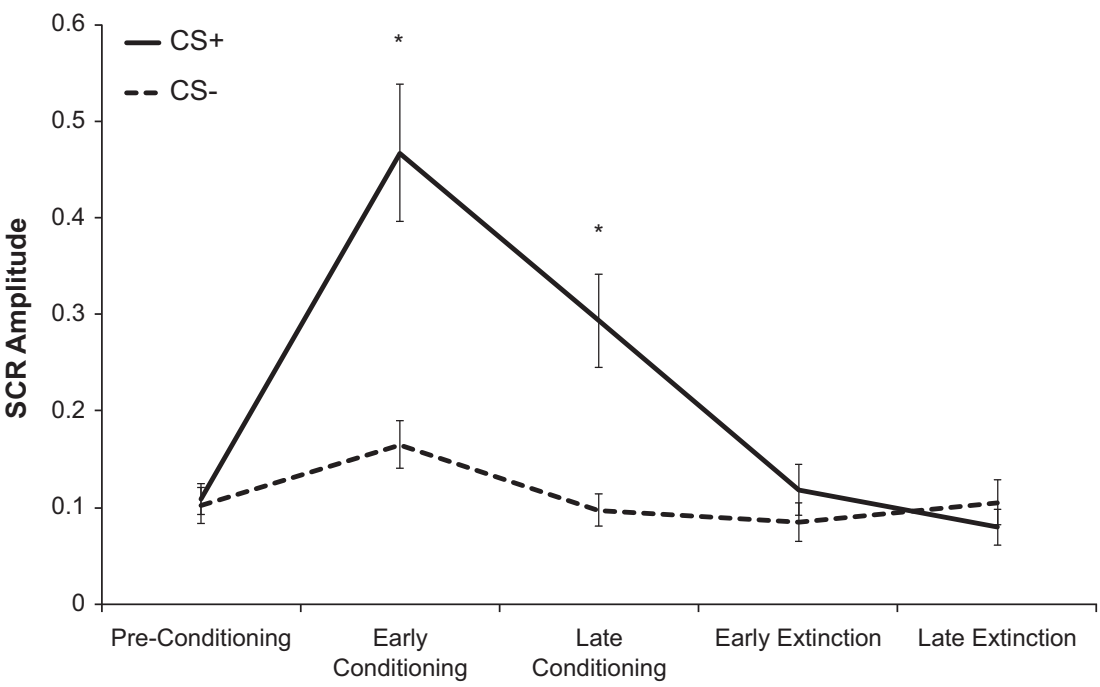

Figure I SCR during fear conditioning task for participants with and without maltreatment exposure. (a) Youths who reported exposure to maltreatment. (b) Youths who reported no exposure to maltreatment. *Significant at $P<0.05$, two-tailed test.

0.28 ) but not sexual abuse (95\% CI: $-0.03,0.20)$ or maltreatment severity (95\% CI: $-0.01,1.13)$.

\section{DISCUSSION}

Disruptions in the acquisition and extinction of conditioned fear may contribute to trauma-related psychopathology (Jovanovich and Ressler, 2010; Lissek and van Meurs, 2015). Although child maltreatment is a potent risk factor for psychopathology, surprisingly little research has examined disruptions in fear conditioning as a potential mechanism in this association among children. In the current study, maltreated children exhibited blunted SCR to threat cues during fear conditioning and failed to exhibit a distinct SCR to threat and safety cues during early conditioning. Children with maltreatment exposure had reduced amygdala and hippocampal volume, which were associated with SCR to threat cues during early fear conditioning. Children with PTSD and externalizing psychopathology exhibited blunted SCR to threat cues during conditioning. This pattern of altered fear conditioning mediated the association of maltreatment with externalizing psychopathology, indicating that disruptions in fear conditioning might serve as a mechanism of increased risk for externalizing problems among maltreated children.

What might explain the absence of threat-safety discrimination among maltreated children during early conditioning? Maltreatment might enhance generalization of conditioned fear to stimuli that resemble threat cues. Color was the only feature that distinguished the threat and safety cue in our paradigm, and maltreated children with trauma histories may have generalized conditioned fear responses from the bell that predicted threat to the bell that signaled safety. Fear generalization is potentially adaptive for children being raised in chronically dangerous environments by 
Table 4 Neural Structure by Trauma Exposure $(N=58)$

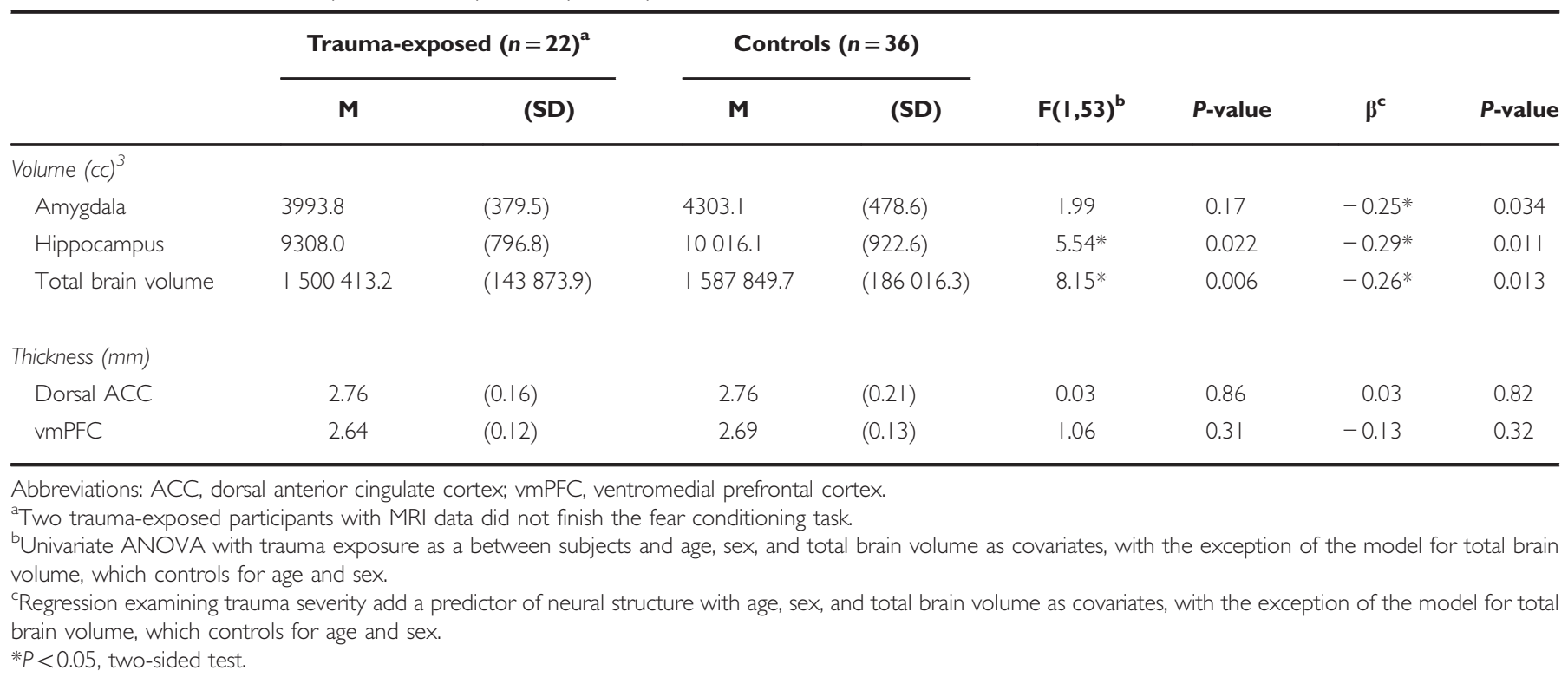

facilitating rapid identification of potential threats. A second possibility is that maltreatment results in a more generalized failure of associative learning, making it difficult for children to predict when the US would occur. This interpretation is consistent with extensive evidence documenting reduced IQ in children who have been maltreated (Koenen et al, 2003). This type of associative learning deficit is associated with heightened contextual anxiety and avoidance (Grillon, 2002). Future research is needed to distinguish between these potential mechanisms.

Maltreated children also exhibited blunted SCR to threat cues during conditioning relative to children with no maltreatment exposure. Projections from the central nucleus of the amygdala to the lateral hypothalamus and then to the brain stem and spinal cord mediate the autonomic component of conditioned fear responses (LeDoux et al, 1988). Excessive release of corticotropin-releasing hormone (CRH) from the hypothalamus following exposure to trauma likely results in downregulation of $\mathrm{CRH}$ receptors in the pituitary and brain stem, leading to reduced production of cortisol and blunted sympathetic nervous system reactivity in response to potential threats, a pattern frequently observed in maltreated children (MacMillan et al, 2009; McLaughlin et al, 2014a). Alternatively, this pattern might be explained by the presence of numbing and dissociative symptoms, which are common among maltreated children (Macfie et al, 2001). Greater research is needed to clarify these pathways.

Amygdala volume was negatively associated with SCR to the threat cue during early fear conditioning and with selfreported fear during conditioning in the total sample. Evidence from rodent and human studies documents the central role of the amygdala in fear conditioning (Johansen et al, 2011; Kim and Jung, 2006). Information about the CS and US converges in the amygdala and produces associative synaptic changes that enhance amygdala responses to the CS. Our findings link amygdala morphology to fear conditioning in children, consistent with evidence from rodents and adult humans suggesting that reduced amygdala volume is associated with enhanced acquisition of conditioned fear
(Hartley et al, 2011; Yang et al, 2008). Hippocampal volume was also negatively associated with SCR to the threat cue during early conditioning. This finding was unexpected, given that the hippocampus is involved only in the acquisition of conditioned fear to complex contextual stimuli but not simple sensory stimuli (Phillips and LeDoux, 1992). Although our conditioning paradigm involved simple sensory cues, it is possible that children associated the US with the environmental context in which fear conditioning occurred; this type of contextual learning involves the hippocampus and has been associated with hippocampal volume in adults (Pohlack et al, 2012). Additional research is necessary to evaluate the role of hippocampal volume in fear conditioning processes across human development.

Given the association of amygdala morphology with early fear conditioning, we would expect maltreated children to have greater SCR to threat cues during early conditioning. Instead, the opposite pattern was observed in the total sample. However, although the interactions of maltreatment with amygdala and hippocampal volume in predicting SCR to threat cues during conditioning were not significant, closer inspection suggested that these association were negative only among non-maltreated children and were reversed (ie, positive) for children with maltreatment exposure. Given our small sample and lack of significant interaction, these findings should be considered preliminary and warrant replication.

Children with externalizing psychopathology exhibited disruptions in fear conditioning that resembled those among maltreated children. This is consistent with previous work documenting poor differential fear conditioning among youths with conduct disorder (Fairchild et al, 2008), difficulty discriminating threatening $v s$ safe social situations among children with externalizing problems and among children with a history of violence exposure (Dodge et al, 1995), and a generalized pattern of blunted sympathetic nervous system reactivity in children with externalizing disorders (Beauchaine et al, 2001; Crowell et al, 2006). Our findings suggest that blunted SCR to threat cues during early 
fear conditioning is one potential mechanism linking maltreatment with externalizing psychopathology in children.

We provide novel evidence for disruptions in fear conditioning among maltreated children. However, several limitations are worth noting. First, our conditioning paradigm did not include an extinction recall phase. Second, we did not assess contingency awareness. However, it is unlikely that group differences in contingency awareness accounted for our findings, because children reported greater fear to the $\mathrm{CS}+$ than $\mathrm{CS}$ - after conditioning and extinction, regardless of maltreatment, suggesting good contingency awareness. Third, the US (ie, a loud noise) was associated with rapid habituation to the CS+, even during the conditioning period, which may have reduced our ability to observe group differences during extinction learning. More potent US (eg, shock) raise ethical and logistical challenges in studies of children. Fourth, we did not assess some potential confounders, such as parent psychopathology, temperament, and pubertal status. Fifth, the associations of neural structure with fear conditioning were modest in magnitude and would not have survived for multiple comparisons, highlighting the importance of replication. Finally, psychopathology was evaluated using questionnaires rather than a clinical interview and anxiety and depression were assessed using childreport only. Replication of our findings in children with externalizing disorders is an important next step.

Child maltreatment is associated with blunted responses to threat cues and poor discrimination between threat and safety cues during fear conditioning in children. Poor threat-safety discrimination might reflect either enhanced fear generalization or a more global deficit in associative learning among maltreated children. Disruptions in fear conditioning might represent a mechanism underlying the development of maltreatment-related externalizing psychopathology in children.

\section{FUNDING AND DISCLOSURE}

This research was supported by a NARSAD Young Investigator Award from the Brain and Behavior Foundation to McLaughlin and grants from the National Institute of Mental Health (K01-MH092526 and R01-MH103291 to KA McLaughlin and K01-MH092555 to MA Sheridan), the National Institute of Child Health and Human Development (U54 HD083091 to the University of Washington's Center on Human Development and Disability), and the Bezos Family Foundation to KA McLaughlin and MA Sheridan. These funders provided support for data collection and analysis. In addition, this research was supported in part by the Intramural Research Program of the National Institutes of Health. Drs. McLaughlin, Sheridan, and Pine have received compensation for activities related to teaching, editing, and clinical care that pose no conflicts of interest. The authors declare no conflict of interest.

\section{REFERENCES}

Achenbach TM (1991). Integrative Guide for the 1991 CBCL/4-18, YSR and TRF Profiles. Department of Psychiatry, University of Vermont: Burlington, VT, USA.

Beauchaine TP, Katkin ES, Strassberg Z, Snarr J (2001). Disinhibitory psychopathology in male adolescents: discriminating conduct disorder from attention-deficit/hyperactivity disorder through concurrent assessment of multiple autonomic states. J Abnorm Psychol 110: 610-624.

Bernstein DP, Ahluvalia T, Pogge D, Handelsman L (1997). Validity of the Childhood Trauma Questionnaire in an adolescent psychiatric population. J Am Acad Child Adolesc Psychiatry 36: 340-348.

Bifulco A, Brown GW, Lillie A, Jarvis J (1997). Memories of childhood neglect and abuse: corroboration in a series of sisters. J Child Psychol Psychiatry 38: 365-374.

Birmaher B, Khetarpal S, Brent D, Cully M, Balach L, Kaufman J et al (1997). The Screen for Child Anxiety Related Emotional Disorders (SCARED): scale construction and psychometric characteristics. J Am Acad Child Adolesc Psychiatry 36: 545-553.

Cacioppa JT, Tassinary LG, Bernston G (2007). Handbook of Psychophysiology. Cambridge University Press: Cambridge, UK.

Craske MG, Waters AM, Bergman RL, Naliboff B, Lipp OV, Negoro $\mathrm{H}$ et al (2008). Is aversive learning a marker of risk for anxiety disorders in children? Behav Res Ther 46: 954-967.

Crowell SE, Beauchaine TP, Gatzke-Kopp L, Sylvers P, Mead HK, Chipman-Chacon J (2006). Autonomic correlates of attentiondeficit/hyperactivity disorder and oppositional defiant disorder in preschool children. J Abnorm Psychol 115: 174-178.

Dodge KA, Petit GS, Bates JE, Valente E (1995). Social informationprocessing patterns partially mediate the effect of early physical abuse on later conduct problems. J Abnorm Psychol 104: 632-643.

Fairchild G, van Goozen SHM, Stollery SJ, Goodyer IM (2008). Fear conditioning and affective modulation of the startle reflex in male adolescents with early-onset or adolescence-onset conduct disorder and healthy control subjects. Biol Psychiatry 63: 279-285.

Fischl B, Dale AM (2000). Measuring the thickness of the human cerebral cortex from magnetic resonance images. Proc Natl Acad Sci 97: 11050-11055.

Glenn CR, Klein DN, Lissek S, Britton JC, Pine DS, Hajcak G (2012). The development of fear learning and generalization in 8-13 year-olds. Dev Psychobiol 54: 675-684.

Grillon C (2002). Associative learning deficits increase symptoms of anxiety in humans. Biol Psychiatry 51: 851-858.

Hanson JL, Chung MK, Avants BB, Shirtcliff EA, Gee JC, Davidson JRT et al (2010). Early stress is associated with alterations in the orbitofrontal cortex: a tensor-based morphometry investigation of brain structure and behavioral risk. J Neurosci 30: 7466-7472.

Hanson JL, Nacewicz BM, Sutterer MJ, Cayo AA, Schaefer SM, Rudolph KD et al (2015). Behavioral problems after early life stress: contributions of the hippocampus and amygdala. Biol Psychiatry 77: 314-323.

Hartley CA, Fischl B, Phelps EA (2011). Brain structure correlates of individual differences in the acquisition and inhibition of conditioned fear. Cereb Cortex 21: 1954-1962.

Hayes AF (2013). Introduction to mediation, Moderation, and Conditional Process Analysis: A Regression-Based Approach. Guilford Press: New York, USA.

Johansen JP, Cain CK, Ostroff LE, LeDoux JE (2011). Molecular mechanisms of fear learning and memory. Cell 147: 509-524.

Jovanovich T, Norrholm SD (2011). Neural mechanisms of impaired fear inhibition in posttraumatic stress disorder. Front Behav Neurosci 5: Article 44.

Jovanovich T, Ressler KJ (2010). How the neurocircuitry and genetics of fear inhibition may inform our understanding of PTSD. Am J Psychiatry 167: 648-662.

Kilpatrick DG, Ruggiero KJ, Acierno R, Saunders BE, Resnick HS, Best CL (2003). Violence and risk of PTSD, major depression, substance abuse/dependence, and comorbidity: results from the National Survey of Adolescents. J Consult Clin Psychol 71: 692-700.

Kim JH, Richardson R (2010). New findings on extinction of conditioned fear early in development: theoretical and clinical implications. Biol Psychiatry 67: 297-303. 
Kim JJ, Jung MW (2006). Neural circuits and mechanisms involved in Pavlovian fear conditioning: a critical review. Neurosci Biobehav Rev 30: 188-202.

Koenen KC, Moffit TE, Caspi A, Taylor A, Purcell S (2003). Domestic violence is associated with environmental suppression of IQ in young children. Dev Psychopathol 15: 297-311.

Kovacs M (1992). Children's Depression Inventory Manual. Multi-Health Systems: North Tonawanda, NY, USA.

Lau JYF, Lissek S, Nelson EE, Lee Y, Roberson-Nay R, Poeth K et al (2008). Fear conditioning in adolescents with anxiety disorders: results from a novel experimental paradigm. J Am Acad Child Adolesc Psychiatry 47: 94-102.

LeDoux JE, Iwata J, Cicchetti P, Reis DJ (1988). Different projections of the central amygdaloid nucleus mediate autonomic and behavioral correlates of conditioned fear. J Neurosci 8: 2517-2529.

Lissek S, van Meurs B (2015). Learning models of PTSD: theoretical accounts and psychobiological evidence. Int J Psychophysiol 98: 594-605.

Macfie J, Cicchetti D, Toth SL (2001). The development of dissociation in maltreated preschool-aged children. Dev Psychopathol 13: 233-254.

MacMillan HL, Georgiades K, Duku EK, Shea A, Steiner M, Niec A et al (2009). Cortisol response to stress in female youths exposed to childhood maltreatment: results of the Youth Mood Project. Biol Psychiatry 66: 62-68.

Matsumoto M, Togashi H, Konno K, Koseki H, Hirata R, Izumi T et al (2008). Early postnatal stress alters the extinction of contextdependent conditioned fear in adult rats. Pharmacol Biochem Behav 89: 247-252.

McCrory EJ, De Brito SA, Sebastian CL, Mechelli A, Bird G, Kelly PA et al (2011). Heightened neural reactivity to threat in child victims of family violence. Curr Biol 21: R947-R948.

McLaughlin KA, Koenen KC, Hill E, Petukhova M, Sampson NA, Zaslavsky A et al (2013). Trauma exposure and posttraumatic stress disorder in a US national sample of adolescents. J Am Acad Child Adolesc Psychiatry 52: 815-830.

McLaughlin KA, Peverill M, Gold AL, Alves S, Sheridan MA (2015). Child maltreatment and neural systems underlying emotion regulation. J Am Acad Child Adolesc Psychiatry 54: 753-762.

McLaughlin KA, Sheridan MA, Alves S, Mendes WB (2014a). Child maltreatment and autonomic nervous system reactivity: identifying dysregulated stress reactivity patterns using the biopsychosocial model of challenge and threat. Psychosom Med 76: $538-546$.

McLaughlin KA, Sheridan MA, Lambert HK (2014b). Childhood adversity and neural development: deprivation and threat as distinct dimensions of early experience. Neurosci Biobehav Rev 47: $578-591$.

Milad MR, Pitman RK, Ellis CB, Gold AL, Shin LM, Lasko NB et al (2009). Neurobiological basis of failure to recall extinction memory in posttraumatic stress disorder. Biol Psychiatry 66: 1075-1082.

Milad MR, Quinn BT, Pitman RK, Orr SP, Fischl B, Rauch SL (2005). Thicnkess of ventromedial prefrontal cortex in humans is correlated with extinction memory. Proc Natl Acad Sci 102: 10706-10711.

Milad MR, Quirk GJ, Pitman RK, Orr SP, Fischl B, Rauch SL (2007). A role for the human dorsal anterior cingulate cortex in fear expression. Biol Psychiatry 62: 1191-1194.

Patwell SS, Duhoux S, Hartley CA, Johnson DC, Jing D, Elliott MD et al (2012). Altered fear learning across development in both mouse and human. Proc Natl Acad Sci 109: 16318-16323.

Phillips RG, LeDoux JE (1992). Differential contributions of amygdala and hippocampus to cued and contextual fear conditioning. Behav Neurosci 106: 274-285.

Pohlack ST, Nees F, Liebscher C, Cacciaglia R, Diener SJ, Ridder S et al (2012). Hippocampal but not amygdalar volume affects contextual fear conditioning in humans. Hum Brain Mapp 33: 478-488.

Pollak SD, Tolley-Schell SA (2003). Selective attention to facial emotion in physically abused children. J Abnorm Psychol 112(3): 323-338.

Shechner T, Britton JC, Ronkin E, Jarcho J, Mash J, Michalska KJ et al (2014a). Fear conditioning and extinction in anxious and non-anxious youth and adults: examining a novel developmentally-appropriate fear conditioning task. Depress Anxiety 32: 277-288.

Shechner T, Hong M, Britton JC, Pine DS, Fox NA (2014b). Fear conditioning and extinction across development: evidence from human studies and animal models. Biol Psychol 100: 1-12.

Sheridan MA, McLaughlin KA (2014). Dimensions of early experience and neural development: deprivation and threat. Trends Cogn Sci 18: 580-585.

Steinberg AM, Brymer MJ, Decker KB, Pynoos RS (2004). The University of California at Los Angeles post-traumatic stress disorder reaction index. Curr Psychiatry Rep 6: 96-100.

Steinberg AM, Brymer MJ, Kim S, Briggs EC, Ghosh Ippen C, Ostrowski SA et al (2013). Psychometric properties of the UCLA PTSD Reaction Index: Part 1. J Trauma Stress 26: 1-9.

Toledo-Rodriguez M, Sandi C (2007). Stress before puberty exerts a sex- and age-related impact on auditory and contextual fear conditioning in the rat. Neural Plast 2007: Article ID 71203.

Walker EA, Unutzer J, Rutter C, Gelfand A, Saunders K, VonKorff M et al (1999). Costs of health care use by women HMO members with a history of childhood abuse and neglect. Arch Gen Psychiatry 56: 609-613.

Yang RJ, Mozhui K, Karlsson RM, Cameron HA, Williams RW, Holmes A (2008). Variation in mouse basolateral amygdala volume is associated with differences in stress reactivity and fear learning. Neuropsychopharmacology 33: 2595-2604.

Supplementary Information accompanies the paper on the Neuropsychopharmacology website (http://www.nature.com/npp) 